\title{
Antimicrobial Susceptibility Pattern of Gram-Negative Bacterial Isolates from Raw Chicken Meat Samples
}

\author{
Neha Gautam ${ }^{1}$, Rojan Poudel ${ }^{1}$, Binod Lekhak ${ }^{2}$, Milan Kumar Upreti ${ }^{1 *}$ \\ ${ }^{1}$ Department of Microbiology, GoldenGate International College, Wisdom Tower, Trikuti Colony, Old \\ Baneshwor, Battisputali, Kathmandu \\ ${ }^{2}$ Central Department of Microbiology, Tribhuvan University, Kritipur, Kathmandu
}

*Corresponding author: Milan Kumar Upreti, Department of Microbiology, Goldengate International College, Kthmandu, Nepal; Email: milanupreti@gmail.com

\begin{abstract}
Objectives: This research aims to study the microbial quality of chicken meat available in retail shop of Kathmandu valley.

Methods: This study was conducted from June to December 2018 in three different districts of Kathmandu Valley. Samples were collected in sterile plastic bags, labeled properly and stored in an icebox and transported to the Food Microbiology laboratory of Golden Gate International College.

During sample preparation, 25 grams of each sample was taken and transferred to sterile flasks containing $225 \mathrm{ml}$ of buffered peptone water. Potential pathogenic Gram-negative bacteria were isolated by using respective selective media and identified by biochemical test. Antibiotic susceptibility profile of isolates was carried out by Kirby-Bauer disc diffusion method according to CLSI 2017 guideline.
\end{abstract}

Results: Of total 81 chicken meat samples processed, $201 \mathrm{Gram}$ negative bacteria were isolated. E. coli (100\%) was the dominant Gram-negative isolates, followed by Citrobacter spp (62.96\%), Pseudomonas spp (40.74\%), Proteus spp (19.75\%), Salmonella spp (16.04\%) and Klebsiella spp (8.64\%) respectively. No any multidrug isolates were detected.

Conclusion: The study showed that the raw chicken meat samples of Kathmandu valley was highly contaminated with Gram negative potential pathogenic bacteria. Antimicrobial resistance pattern shown by the isolates may indicates that there is not overuse of drug in animals and the less chance of risk of increasing antimicrobial resistance.

Key words: Chicken meat, Antimicrobial susceptibility test, Gram negative bacteria

\section{INTRODUCTION}

Poultry meat is the combination of muscle tissue, attached skin, connective tissue, and edible organs, comprising about two-thirds of the total meat production in the world (Ruban et al. 2010). Chicken meat is considered as a healthy food, due to high protein content and reduced fat content, as well as a higher proportion of polyunsaturated fatty acids (PUFA), when compared to other species meats (Riovanto et al. 2012). In Asian countries, chicken meat was periodically included in the diets of consumers in

Date of Submission: November 10, 2019

Published Online: December, 2019 the past. However, with the rapid economic growth and globalization of the food industry, the amount of meat production and consumption has grown rapidly in recent years (Nam et al. 2010).

Due to its high percentages of nitrogenous compounds of various degrees of complexity, high moisture, abundant supply of minerals, accessory growth factors and some fermentable carbohydrates (glycogen) of a suitable $\mathrm{pH}$, meat are regarded as a perfect culture medium for most of the microorganisms (Holck et al. 2017). The hygiene often fails during slaughtering,

Date of Acceptance: December 4, 2019

DOI: https:/ / doi.org/10.3126/tujm.v6i0.26590 
scalding, evisceration, plucking, bleeding, washing, and rinsing, and increase the health risk associated with the consumption of this meat (Coulibaly et al. 2010). Similarly, animals can also become infected from water or food contaminated with wastes of human or animal origin or with human carrier workers (Da Silva et al. 2011). One of the possible ways of entry of various microbes could be the handling of meat and meat products by adopting improper hygienic measures during handling and processing (Kiranmayi et al. 2011). It has been pointed out that during slaughter, dressing, and cutting, microorganisms come chiefly from the exterior of the animal and its intestinal tract but more are added from knives, cloths, air, workers, carts, boxes, and equipment in general (Bhandare et al. 2007). Escherichia, Enterobacter, Klebsiella, Proteus, Citrobacter, Serratia, Salmonella, Shigella and Yersinia, are among the most important causes bacterial infections in humans (Jarzab et al. 2011).

Antibiotics are often used for therapy of infected humans and animals as well as for prophylaxis and growth promotion of food producing animals. Many findings suggest that inadequate selection and abuse of antimicrobial may lead to resistance in various bacteria sand make the treatment of bacterial infections more difficult (Kolar et al. 2001).

Poor personal hygiene is prime cause of food borne illness. Hand washing is the crucial measure to prevent propagation of diseases and to cut back the transmission of bacteria among individuals and to food as well (Lambrechts et al. 2014). Resistant bacteria are transmitted to human through direct contact with animal, by exposure to animal manure, through consumption of uncooked meat, and through contact with meat surfaces (Marshall and Levy 2011). The expansion of antibiotic resistance in bacteria is also an emerging public health hazard due to the compromised efficacy in the treatment of infectious diseases (Helmy et al. 2017).

\section{MATERIALS AND METHODS}

Study was conducted from June to December 2018. Random, purposive sampling was fconducted for the collection of sample to study. A total of 81 raw chicken meat samples were collected from different retail meat shops at Kathmandu, Lalitpur and Bhaktapur districts.

Samples were collected in sterile plastic bags. Samples collected from different butcher shops at Lalitpur,
Kathmandu and Bhaktapur districts were labeled properly and stored in an icebox until delivery and transported to the Food Microbiology laboratory of Golden Gate International College. Raw chicken meat was collected from different meat shops and studied. Packaged, cold stored or chemically treated meat was not collected (Da Silva et al. 2013).

During sample preparation, 25 gram of each sample was taken and transferred to sterile flasks containing $225 \mathrm{ml}$ of buffered peptone water. Samples were being homogenized using a meat grinder under aseptic condition and kept in rotatory shaker at $80 \mathrm{rpm}$ for 30 minutes (Shrestha et al. 2017).

For the enrichment, $1 \mathrm{ml}$ of the sample from the flask was suspended in $9 \mathrm{ml}$ of Selenite Broth and incubated for 24 hours at $37^{\circ} \mathrm{C}$ (Shrestha et al. 2017). Loopful of sample from Selenite broth was streaked on Xylose Lysine Deoxycholate (XLD) agar plates, plates were then incubated at $37^{\circ} \mathrm{C}$ for 24 hours (Da Silva et al. 2013).

Plates were examined for presence of typical Salmonella colonies. Salmonella colonies may appear pink colonies with black center (Shrestha et al. 2017). Isolated colony was taken and streaked in Nutrient agar and incubated for 24 hours at $37^{\circ} \mathrm{C}$. Isolated colony from NA was transferred to Triple Sugar Iron Agar (TSIA) and was incubated at $37^{\circ} \mathrm{C}$ for 24 hours for the confirmation of Salmonella (Shrestha et al. 2017). For the enrichment, $1 \mathrm{ml}$ of the sample from the flask was suspended in $9 \mathrm{ml}$ of Nutrient Broth and incubated for 24 hours at $37^{\circ} \mathrm{C}$ (Shrestha et al. 2017). Loopful of sample from Nutrient Broth was streaked on M-endo agar. The plates were incubated at $37{ }^{\circ} \mathrm{C}$ for 24 hours. Colonies exhibiting metallic sheen on M-endo agar was sub cultured on NA to obtain pure culture. Pure cultures were confirmed to be $E$. coli by biochemical tests (IMViC, TSI, O/F and Urease) (Da Silva et al. 2013).

Isolation and identification of Pseudomonas spp. was done by streaking loopful sample from NA to cetrimide agar plate. Colonies showing yellowish green color was tested for conformation of Pseudomonas (Estepa et al. 2015).

Colonies showing pink color in Mac conkey agar plate were conformed as Gram negative isolates and species were confirmed by following CLSI guidelines 2017.

Testing for antibiotic sensitivity was done by the 
Modified Kirby-Bauer Disc diffusion method by using Muller Hilton Agar. The bacterial suspensions made in normal saline were compared with 0.5 McFarland solutions and swabbed on MHA plate using sterile cotton swab. Antibiotic disc were then placed on the swabbed MHA plate. After overnight incubation at $37^{\circ} \mathrm{C}$ the zone of inhibition was observed around the antibiotic disc. Using the CLSI guidelines, the

\section{RESULTS}

Of the total of 201 potential bacterial pathogens isolates, meat samples from the Kathmandu district show the highest contamination and samples from the Lalitpur least as shown in Table 1.

Table 1: Percentage occurrence of bacterial pathogens in Kathmandu valley

\begin{tabular}{lcc}
\hline Districts & Number & Percentage \\
\hline Kathmandu & 84 & 41.79 \\
Bhaktapur & 61 & 30.34 \\
Lalitpur & 56 & 27.86 \\
\hline
\end{tabular}

Among 81 samples examined from three different districts, Gram negative bacterial isolates identified were E. coli, Salmonella spp, Proteus spp, Citrobacter spp, Pseudomonas spp, and Klebsiella spp. respectively. Among them, E. coli was isolated from all the samples 81 (100\%), Salmonella spp. from 13 (16.04\%) samples, Pseudomonas spp. from 33 (40.74\%) samples, Proteus

spp. from $16(19.75 \%)$ samples, Klebsiella spp. from 7 (8.64\%) samples and Citrobacter spp. from 51 (62.96\%) samples as shown in Table 2. Among the 27 meat samples collected from each district, E. coli was isolated from all the samples, whereas Klebsiella was not isolated from sample of Bhaktapur and salmonella from sample of Lalitpur district.

Table 2: Percentage occurrence of the isolated bacteria from chicken sample

\begin{tabular}{lcc}
\hline Bacteria & No. of isolates & Percentage \\
\hline E. coli & 81 & 100.00 \\
Salmonella ssp & 13 & 16.04 \\
Pseudomonas ssp & 33 & 40.74 \\
Proteus ssp & 16 & 19.75 \\
Klebsiella ssp & 7 & 8.64 \\
Citrobacter ssp & 51 & 62.96 \\
\hline
\end{tabular}

Antibiotics susceptibility testing showed all isolates of E. coli were resistant to Ampicillin and sensitive to

Amikacin, Gentamicin, Ceftriaxone and Cotrimoxazole (Table 3).

Table 3: Sensitivity pattern of $E$. coli $(\mathrm{n}=81)$

\begin{tabular}{lcc}
\hline Antibiotics & Sensitive (\%) & Resistant (\%) \\
\hline Ampicillin & - & $81(100)$ \\
Ciprofloxacin & $78(96.29)$ & $3(3.71)$ \\
Gentamicin & $81(100)$ & - \\
Chloramphenicol & $80(98.76)$ & $1(1.24)$ \\
Tetracycline & $9(11.11)$ & $72(88.89)$ \\
Cotrimoxazole & $81(100)$ & - \\
Amikacin & $81(100)$ & - \\
Ceftriaxone & $81(100)$ & - \\
\hline
\end{tabular}

All the isolates of Salmonella spp. isolates were sensitive

to Cotrimoxazole, Ciprofloxacin, Amikacin and 
Table 4: Sensitivity pattern of Salmonella spp. $(n=13)$

\begin{tabular}{lcc}
\hline Antibiotics & Sensitive (\%) & Resistant (\%) \\
\hline Ampicillin & - & $13(100)$ \\
Ciprofloxacin & $13(100)$ & - \\
Gentamicin & $11(84.62)$ & $2(15.38)$ \\
Chloramphenicol & $9(69.23)$ & $4(30.77)$ \\
Tetracycline & $3(23.07)$ & $10(76.93)$ \\
Cotrimoxazole & $13(100)$ & - \\
Amikacin & $13(100)$ & - \\
Ceftriaxone & $13(100)$ & - \\
\hline
\end{tabular}

All the isolates of Pseudomonas spp. isolates were subjected to AST and found sensitive to Gentamicin,
Ciprofloxacin, Amikacin, Ceftriaxone and Cotrimoxazole and resistant to Ampicillin (table 5).

Table 5: Sensitivity pattern of Pseudomonas spp. $(\mathrm{n}=33)$

\begin{tabular}{lcc}
\hline Antibiotics & Sensitive (\%) & Resistant (\%) \\
\hline Ampicillin & - & $33(100)$ \\
Ciprofloxacin & $33(100)$ & - \\
Gentamicin & $33(100)$ & - \\
& $8(24.24)$ & $25(75.76)$ \\
Chloramphenicol & $10(30.30)$ & $23(69.70)$ \\
Tetracycline & $22(66.67)$ & $11(33.33)$ \\
Cotrimoxazole & $33(100)$ & - \\
Amikacin & $33(100)$ & - \\
Ceftriaxone & & \\
\hline
\end{tabular}

All the isolates of Citrobacter spp isolates were found to be sensitive to Chloramphenicol, Ciprofloxacin, Amikacin, Gentamicin and Ceftriaxone. All the isolates of Proteus spp. were sensitive to Gentamicin, Cotrimoxazole, Amikacin and Ceftriaxone and resistant to Tetracycline. All the isolates of Klebsiella spp were sensitive to Amikacin and Ceftriaxone. No any MDR isolates were identified.

\section{DISCUSSION}

Out of 81 samples collected from different meat shops, a total of 201 potential pathogenic Gram negative bacterial isolates were detected among which, all samples showed growth with multiple isolates. Similar result were reported from other studies conducted in North East India, E. coli (98\%), Citrobacter (52\%), and Salmonella (20\%) (Sharma 2012).

In this study all the samples showed presence of E. coli, which is very high as compared to previous study (4.8\%) by Shrestha et al. 2017 and Thanigaivel and Anandhan 2015 (70\%). The reason behind this huge difference could be due to the use of contaminated water during slaughtering, washing and in other handling processes, and also due to E. coli being common inhabitants of animal and human guts (Cabral 2010). As this study does not focus on the water quality of the water used in the meat shops and the parameters on the water quality are not studied either. Total of $62.96 \%$ of occurrence of Citrobacter spp was seen, which is less as compared to result presented from other studies, (Shrestha et al. 2017) i.e. 44.7\% and (Gwida 2014) in Egypt which is $13.3 \%$. The higher percentage of C. freundii presence in foods can be ascribed to secondary contamination (Ryan et al. 2004; Raphael and Riley 2017).

Thirteen $(16.04 \%)$ isolates were detected as Salmonella spp which is much lower than the result presented in other similar studies from Egypt, Azez (2013) i.e. 44\% and Nigeria i.e 33.33\% (Adeyanju and Ishola 2014).

About 41 \% (33) isolates were Pseudomonas spp. which is much higher than $1.9 \%$ reported (Shrestha et al. 2017) in Bharatpur, Nepal and 10\% (Thanigaivel and Anandhan 2015) in India but somewhat similar to $46.66 \%$ found in Dharan (Bantawa et al. 2018). P. aeruginosa is one of the most relevant opportunistic human pathogens, although there are also reports that show clinical cases caused by environmental Pseudomonas, such as $P$. mendocina and P. fulva (Nseir et al. 2011). Differences in prevalence rates from this study to another might be attributed to the unhygienic processing and poor sanitation of meat shops. It showed that meat retailers were found to be unaware of the basic requirements of 
basic guidelines regarding meat (Almuzara et al. 2010 and Seok et al. 2010).

Percentage of Proteus spp. isolates were more similar to study by Shrestha et al. (2017) but lower than study by Al-Mutairi (2011) from Saudi Arabia. Proteus spp. is considered as an indicator of contamination of meat during any of the processing, handling, storage stages. If the optimal condition for the isolated Proteus were existed, typical cases of food poisoning, urinary infection and other Proteus related human illnesses could happen due to rapid proliferation of the pathogen (Al-Mutairi 2011).

Klebsiella spp had least occurrence as compared to other isolates i.e.7 (8.64\%), which is very less as compared to the result shown (Kim et al. 2005) in Oklahoma which is $30 \%$ but similar to Al-Mutairi 2011 (10.66\%). Klebsiella spp is a colonizing opportunistic pathogen of humans and animals, and a common contaminant of retail meat (Kim et al. 2005). In animals, Klebsiella spp. causes disease in cows, horses, and other animals (Bersisa et al. 2019 and Ewers et al. 2014). In humans, Klebsiella spp. frequently colonizes the gut and sporadically causes extra intestinal infections (Podschun and Ullmann 1998).

All E. coli isolates were resistance to Ampicillin, $88.89 \%, 3.71 \%$ and $1.24 \%$ were resistant to Tetracycline, Ciprofloxacin and Chloramphenicol respectively. All of isolates were sensitive to Cotrimoxazole, Amikacin, Gentamicin and Ceftriaxone followed by $98.76 \%$, $96.29 \%$ and $11.11 \%$ sensitivity to Chloramphenicol, Ciprofloxacin and Tetracycline respectively. In the study carried at Nigeria $83 \%$ of E. coli isolates were resistant to Tetracycline, $89 \%$ resistant to Cotrimoxazole and $28.3 \%$ resistant to Gentamicin (Adeyanju and Ishola 2014). Thanigaivel et al. 2015 in India reported $26 \%$ resistivity of E. coli to Tetracycline. Somda et al. 2018 in Burkina Faso, reported 100\% sensitivity to Ciprofloxacin, Gentamicin, and Chloramphenicol. However, resistance was observed with Ceftriaxone (10.71\%), Ampicillin (42.86\%), and tetracycline (64.3\%).

A potential health hazard to consumers can be expected from resistant bacteria. If the organism is resistant to antibiotics, then initial treatment may be ineffective both in man and animals and an alternative treatment need to be applied (cotterill et al. 1977).

Total of 13 isolates of Salmonella spp., 100\% were sensitive to Ciprofloxacin, Cotrimoxazole, Amikacin, and Ceftriaxone and 100\% were resistant to Ampicillin. Only $84.62 \%, 69.23 \%$, and $23.07 \%$ sensitivity were shown towards Gentamicin, Chloramphenicol and Tetracycline respectively. The study conducted in Egypt (Moawad et al. 2017) reported that $87 \%$ and $40 \%$ Salmonella spp were resistant to Ampicillin and Tetracycline, 53\% sensitive to Chloramphenicol and $67 \%$ sensitive to Ciprofloxacin.

Similarly, all Pseudomonas spp. isolates were found sensitive towards Ciprofloxacin, Gentamicin, Amikacin and Ceftriaxone and completely resistant to Ampicillin. Among which $24.24 \%, 30.30 \%$ and $66.67 \%$ were sensitive to Chloramphenicol, Tetracycline and Cotrimoxazole respectively. According to result reported by Estepa et al. 2015 in Spain, Pseudomonas spp. showed 100\% sensitivity to Amikacin, Gentamicin, Kanamicin, and Tobramycin.

Citrobacter spp. showed sensitivity towards many antibiotics such as, Ciprofloxacin, Gentamicin, Chloramphenicol, Amikacin and Ceftriaxone. Liu et al. 2017 in China reported that all Citrobacter spp. isolates were sensitive to Amikacin, $66.67 \%$ were resistant to Ampicillin, $16.67 \%$ resistivity to Ciprofloxacin, $5.56 \%$ resistivity to Gentamicin, $44.45 \%$ resistivity to Tetracycline and $27.78 \%$ resistivity to Cotrimoxazole.

All Proteus spp. isolates were found sensitive towards Gentamicin, Amikacin, Cotrimoxazole and Ceftriaxone and resistant to Tetracycline. Shrestha et al. 2017 in Bharatpur, Nepal reported $11.7 \%$ of Proteus spp. resistant to Ciprofloxacin.

All Klebsiella spp. isolates were found sensitive towards Amikacin and Ceftriaxone. They showed $14.29 \%, 85.71 \%, 71.43 \%, 42.86 \%, 57.14 \%$ and $71.43 \%$ sensitivity to Ampicillin, Ciprofloxacin, Gentamicin, Chloramphenicol, Tetracycline and Cotrimoxazole respectively. According to the report of Kim et al. 2005 , the study conducted in Oklahoma, 100\% of Klebsiella isolates were found resistant to Ampicillin, Tetracycline and also Ceftriaxone. Davis et al. (2015) also reported $100 \%$ resistivity towards Ampicillin and $100 \%$ sensitivity to Amikacin.

Antimicrobial are used even in the absence of illness to prevent diseases when animals are susceptible to infection (Turtura et al. 1990). In slaughterhouse, resistant strains from the gastrointestinal tract may infect chicken carcasses and, as a result, chicken meats are often related to antimicrobial-resistant 
microorganisms (Reza et al. 2014). Therefore, these antimicrobial-resistant bacteria from poultry can infect humans directly and indirectly with food. Though rarely, these resistant bacteria may colonize in the human gastrointestinal tract and may also transfer resistance bacteria to human endogenous flora (Reza et al. 2014). However, the rate of MDR for all the isolates was nil whereas, $79.6 \%$ prevalence of MDR bacteria was found in chicken meat in Bharatpur (Shrestha et al. 2017).

Poor hygienic practices during slaughtering and marketing of meat are one of the major contributing factors for unsafe meat in Nepal (Joshi et al. 2003). Slaughtering animals in open and unhygienic places, use of dirty water during slaughtering process, and selling meat in open and non-refrigerated places are some of the unhygienic practices being used by the sellers (Sharma 2012).

\section{CONCLUSION}

Out of 81 samples, all the samples showed the presence of potential pathogenic bacteria with $E$. coli being the dominant. Antibiogram studies of all isolates against 8 different antibiotics showed that majority of isolates were sensitive. And rate of MDR was nil for each isolate. Among the three districts, Lalitpur showed comparatively satisfactory hygienic condition in relative to Bhaktapur and Kathmandu. Slaughtering of animals in unhygienic place, not maintaining the good hygiene practices during cutting, handling, transportation results to the poor quality of the meat and furthermore, use of polluted water contaminate the meat with different microorganism which can be harmful for the health of the consumers and which can increase the risk of food-borne illness and epidemic diseases

\section{ACKNOWLEDGEMENTS}

Sincerely thankful to all people who were very helpful during the sample collection for this study and Goldengate Microbiology Faculty and laboratory members as well as to my friends for their help, encouragement and exchange of knowledge.

\section{CONFLICT OF INTEREST}

The authors declare no conflict of interest.

\section{REFERENCES}

Adeyanju GT and Ishola O (2014). Salmonella and Escherichia coli contamination of poultry meat from a processing plant and retail markets in
Ibadan, Oyo State, Nigeria. Springer Plus 3: 139.

Almuzara MN, Vazquez M, Tanaka N, Turco M, Ramirez MS, Lopez EL, Pasteran F, Rapoport M, Procopio A and Vay CA (2010). First case of human infection due to Pseudomonas fulva, an environmental bacterium isolated from cerebrospinal fluid. J Clin Microbiol 48: 660-664.

Al-Mutairi MF (2011). The incidence of enterobacteriaceae causing food poisoning in some meat products. Advance Journal of Food Science and Technology 3(2): 116-121

Bantawa K, Rai K, Subba Limbu D and Khanal H (2018). Food-borne bacterial pathogens in marketed raw meat of Dharan, Eastern Nepal. BMC Res Notes 1 (1): $1-5$

Bersisa A, Tulu D and Negera C (2019). Investigation of bacteriological quality of meat from abattoir and butcher shops in Bishoftu, Central Ethiopia. International Journal of Microbiology 1: 1-8.

Bhandare SG, Sherikarv AT, Paturkar AM, Waskar VS and Zende RJ (2007). A comparison of microbial contamination on sheep/goat carcasses in a modern Indian abattoir and traditional meat shops. Food Control 18: 854-868.

Cabral Joao PS (2010). Water Microbiology. Bacterial pathogens and water. Int J Environ Res Public Health 7(10): 3657-3703.

Cotterill OJ, Glauert HP and Russell WD (1977). Microbial counts and thermal resistance of Salmonella oranienburg in ground turkey meat. Poult Sci 56(6):1889-92.

Da Silva DCF, De Arruda AMV and Gonçalves AA (2017). Quality characteristics of broiler chicken meat from free-range and industrial poultry system for the consumers. J Food Sci Technol 54(7): 1818-1826.

Da Silva N, Taniwaki MH, Junqueira VCA, Silveira NFA, do Nascimento MS and Gomes RAR (2013). Microbiological examination methods of food and water: a laboratory manual. London: Taylor \& Francis Group

Estepa V, Rojo-Bezares B, Torres C and Saenz Y (2015). Genetic lineages and antimicrobial resistance in Pseudomonas spp. isolates recovered from food samples. Food-borne Pathogens and Disease 1: 1-6. 
FAO (2009). Food and Agriculture Organization article on egg. Food and Agriculture Organization of United Nations 2004- 03-07.

Helmy MO and Kashef TM (2017). Different phenotypic and molecular mechanisms associated with Multidrug resistance in Gram-negative clinical isolates from Egypt. Infect Drug Resist 10: 479-498.

Holck A, Axelsson L, McLeod A, Rode TM and Heir E (2017). Health and safety considerations of fermented sausages. Hindawi Journal of Food Quality 1: 1- 25.

Joshi DD, Maharjan M, Johansen MV, Willingham AL and Sharma M (2003). Improving meat inspection and control in resource-poor communities: the Nepal example. Acta Tropica 87 (1): 119-127.

Kiranmayi CB, Krishnaiah N, Subhashini N, Amaravathi P, Maheswari M and Ramya P (2011). PCR analysis of mutton and chicken samples for the presence of Shiga toxigenic E. coli. Arch Clin Microbiol 2: 2-4

Kolar M, Urbanek K and Latal T (2001). Antibiotic selective pressure and development of bacterial resistance. Int J Antimicrob Agents 17: 357-63.

Lambrechts AA, Human IS, Doughari JH and Lues JFR (2014). Bacterial contamination of the hands of food handlers as indicator of hand washing efficacy in some convenient food industries. Pak J Med Sci 30(4): 755-758.

Liu X, Jayasena DD, Jung Y, Jung S, Kang BS, Heo KN, Lee JH, and Jo C (2012). Differential proteome analysis of breast and thigh muscles between Korean native chickens and commercial broilers. Asian Australas J Anim Sci 25: 895-902.

Marshall BM and Levy SB (2011). Food animals and antimicrobial: impacts on human health. Clin Microbiol Rev 24: 718-33.

Moawad AA, Hotzel H, Awad O, Tomaso H, Neubauer $\mathrm{H}$ and Hafez HM (2017). Occurrence of Salmonella enterica and Escherichia coli in raw chicken and beef meat in northern Egypt and dissemination of their antibiotic resistance markers. Gut Pathog 9(1): 57

Podschun R and Ullmann U (1998). Klebsiella spp. as nosocomial pathogens: epidemiology, taxonomy, typing methods, and pathogenicity factors. Clin Microbiol Rev 11: 589-603.
Reza T, Mehdi K, Faham K and Mohammad RF (2014). Multiple antimicrobial resistance of Escherichia coli isolated from chickens in Iran. Veterinary Medicine International Article ID 491418, 4 pages

Riovanto R, Marchi M, Cassandro M and Penasa $M$ (2012). Use of near infrared transmittance spectroscopy to predict fatty acid composition of chicken meat. Food Chem 134(4): 2459-2464.

Ruban SW, Thiyageeswaran M and Sharadha R (2010). Isolation and identification of Salmonella spp. from retail chicken meat by polymerase chain reaction. Int J Microbiol Res 1(3): 106-109.

Ryan KJ and Ray CG (2004). Sherris Medical Microbiology, $4^{\text {th }}$ ed. McGraw Hill $p 370$.

Sharma Bajagai Y (2012). Food safety regulation in Nepal and issues in regulation of meat safety. Available at https:/ / www.foodandenvironment. com/2012/11/food-safety-regulation-in-nepal. html

Seok Y, Shin H, Lee Y, Cho I, Na S, Yong D, Jeong SH and Lee K (2010). First report of bloodstream infection caused by Pseudomonas fulva. J Clin Microbiol 48: 2656-2657.

Shrestha A, Bajracharya AM, Subedi H, Turha RS, Kafle S, Sharma S and Chaudhary DK (2017). Multidrug resistance and extended spectrum beta lactamase producing Gram negative bacteria from chicken meat in Bharatpur Metropolitan, Nepal. BMC Res Notes 10(574): 1-5

Somda NS, Traorore O, Bonkoungou O JI, Bassole IHN, Traore Y, Barro N and Savadogo A (2017). Serotyping and antimicrobial drug resistance of Salmonella isolated from lettuce and human diarrhea-samples in Burkina Faso. African Journal of Infectious Diseases 11(2): 024-030.

Thanigaivel G and Anandhan AS (2015). Isolation and characterization of microorganism from raw meat obtained from different market places in and around chennai. Journal of Pharmaceutical, Chemical and Biological Sciences 3(2): 295-301

Turtura GC, Massa S and Ghazvinizadeh H (1990). Antibiotic resistance among coliform bacteria isolated from carcasses of commercially slaughtered chickens. International Journal of Food Microbiology 11(3-4): 351-354. 